\title{
ORIGINAL
}

\section{BARRERAS A LA INTRODUCCIÓN DE UNA AGENCIA EVALUADORA PARA INFORMAR LA FINANCIACIÓN O LA DESINVERSIÓN DE PRESTACIONES SANITARIAS DEL SISTEMA NACIONAL DE SALUD $(*)$}

\author{
Juan José Artells (1), Salvador Peiró (2,3) y Ricard Meneu (3).
}

(1) Fundación Salud, Innovación y Sociedad. Barcelona.

(2) Centro Superior de Investigación en Salud Pública (CSISP-FISABIO). Red de Investigación en Servicios de Salud en Enfermedades Crónicas (REDISSEC). Valencia. España.

(3) Fundación Instituto de Investigación en Servicios de Salud.

(*) El proyecto fue financiado por la Fundación Salud, Innovación y Sociedad, dependiente de Novartis Farmacéutica. Los miembros del panel no recibieron ningún tipo de remuneración por su participación en el mismo.

Conflicto de intereses: JJA estaba contratado por la Fundación Salud, Innovación y Sociedad en el momento de la realización del panel. Los participantes en el panel no comparten necesariamente el contenido de este manuscrito ni las opiniones expresadas por el conjunto del panel.

\begin{abstract}
RESUMEN
Fundamentos: A diferencia de lo que ocurre en otros países, el Sistema Nacional de Salud (SNS) español no ha incorporado el análisis coste-efectividad en la toma de decisiones de las tecnologías y prestaciones que integran cartera de servicios, ni ha desarrollado los organismos de evaluación capaces de desarrollar esta función. El objetivo de este trabajo es identificar las dificultades, obstáculos y limitaciones que han contribuido a estas carencias.

Métodos: Reunión de 14 expertos, en julio de 2012, estructurada siguiendo procedimientos adaptados de las técnicas de tormenta de ideas, grupo nominal y método de consenso Rand.

Resultados: El panel propuso 77 ítems como posibles dificultades, obstáculos o limitaciones para el establecimiento de un "ente" oficial e independiente con capacidad para informar sobre la financiación y desinversión selectiva de las prestaciones del SNS. Estos ítems se centraron en: 1) ausencia de voluntad política de los gobiernos estatal y autonómicos para introducir el análisis coste-efectividad y la falta de independencia y transparencia de los procesos de evaluación, 2) la tensión entre un sistema sanitario descentralizado y la actividad evaluadora que tiene importantes economías de escala, 3) dificultades técnicas de los procesos evaluadores y de su capacidad para influir en la toma de decisiones, y 4) rechazo social y profesional a la exclusión de prestaciones cuando es percibida como indiscriminada.
\end{abstract}

Conclusiones: Aunque existe un variado número y tipo de obstáculos en el desarrollo de la capacidad del SNS para incorporar o excluir prestaciones en función de la evaluación de su efectividad y efíciencia, los expertos sitúan en el plano más político del sistema sanitario las principales dificultades para avanzar en este terreno (voluntad política, transparencia, gobernanza).

Palabras clave: Política sanitaria. Evaluación de tecnologías. Toma de decisiones. Racionamiento. Procesos grupales.

\section{Correspondencia}

Salvador Peiró

Centro Superior de Investigación en Salud Pública (CSISP-FISABIO).

Avda Cataluña 21, 46020 Valencia, España

Correo electrónico: peiro_bor@gva.es

\section{ABSTRACT \\ Barriers for Introducing a Health Technology Assessment Organization to Inform Health Care Decision-Making in the Spanish National Health System}

Background: To identify difficulties, obstacles and limitations to establish an organizational structure devoted to the evaluation of healthcare technologies for incorporation, maintenance or removal from the services portfolio of the Spanish National Health System (sNHS).

Methods: Panel of 14 experts, structured according to processes adapted from brainstorming, nominal group, and Rand consensus method techniques.

Results: The panel proposed 77 items as potential obstacles to the establishment of an official and independent "agency" able to inform on sNHS healthcare benefits funding or selective disinvestment. These items were focused on: 1) lack of political motivation to introduce the cost-effectiveness analysis from the state and regional governments and lack of independence and transparency of the evaluation processes, 2) the tension between a decentralized health system and evaluation activities with significant scale economies, 3) technical difficulties of the evaluation processes, including their ability to influence decision making and 4) social and professional refusal to the exclusion of healthcare benefits when it is perceived as indiscriminate.

Conclusion: Although there is a different number and type of obstacles for developing the capacity of the sNHS to include or exclude healthcare benefits based on the evaluation of their effectiveness and efficiency, experts place in the political arena (political motivation, transparency, governance) the main difficulties to advance in this field.

Keyword: Health Policy. Technology Assessment. Decision Making, Organizational. Health Care Rationing. Group Processes. 


\section{INTRODUCCIÓN}

Actualmente existe una importante experiencia internacional ${ }^{1-7}$ sobre las ventajas de seleccionar y financiar las prestaciones sanitarias de cobertura pública con arreglo a criterios explícitos y métodos contrastados que, además de su efectividad, seguridad y calidad (las tres primeras "garantías"), tengan en cuenta su coste-efectividad y su impacto presupuestario (la llamada cuarta "garantía" o cuarta "valla") ${ }^{8}$. También existe cierto consenso sobre la necesidad de utilizar criterios y métodos similares para las estrategias de "desinversión", la desfinanciación selectiva de prácticas clínicas de dudoso valor (o de menor valor relativo que otras de similar efectividad) y la liberación de esos recursos para financiar otros procedimientos con mejor perfil de efectividad, seguridad y coste-efectividad ${ }^{9}$.

Sin embargo, las estrategias de financiación (y de no financiación) selectiva de servicios y prestaciones no han prosperado en el Sistema Nacional de Salud (SNS) ${ }^{10,11}$. Al menos no más allá de excluir de la cobertura algunos fármacos con alto potencial de abuso (para la obesidad, la deshabituación tabáquica o la disfunción eréctil), algunos medicamentos de dudosa eficacia y/o balance riesgo/beneficio poco favorable (programas PROSEREME) ${ }^{12} \mathrm{o}$, más recientemente, algunos fármacos para "síntomas menores" "13. En ninguno de estos casos se consideraron criterios de coste-efectividad y, en la experiencia más reciente, las "metodologías" utilizadas para la selección de fármacos a excluir - propuesta de varias comunidades autónomas (CCAA) - parecen escasamente científicas y poco reproducibles.

En el SNS, el análisis coste-efectividad (CEA) continúa sin ser exigido para la inclusión o exclusión de tecnologías, medicamentos, procedimientos o servicios en la cartera de prestaciones $\mathrm{y}$, aunque tanto el Ministerio como las CCAA encargan informes de evaluación de tecnologías y guías de práctica (con o sin inclusión de evaluaciones económicas), no existe una estructura organizativa formal que evalué estas prestaciones con la función explícita de informar la toma de decisiones sobre su inclusión/exclusión en la cartera de servicios del SNS. Esta situación es especialmente llamativa en el contexto de una crisis económica profunda y muy duradera que hace más visible y más perentoria la necesidad de priorizar actuaciones, minimizar el despilfarro y mejorar la eficiencia del SNS.

El objetivo de este trabajo es identificar las dificultades, obstáculos y limitaciones para establecer una estructura organizativa "independiente" dedicada a la evaluación de las prestaciones sanitarias (tecnologías, medicamentos, intervenciones, procedimientos, etc.) para su incorporación, mantenimiento o desinversión de la cartera de servicios del SNS así como ordenarlos en función de su importancia relativa.

\section{SUJETOS Y MÉTODOS}

Diseño. Reunión de expertos, estructurada siguiendo procedimientos adaptados de las técnicas de brainstorming ${ }^{14}$, grupo nominal 14-16 y método de consenso Rand $^{16,18}$.

Ámbito/lugar. La reunión se realizó el 7 de julio del 2012 en los locales de la Agència d'Informació, Avaluació i Qualitat en Salut (AIAQS), una empresa pública dependiente del Departament de Salut de la Generalitat de Catalunya, que facilitó los medios necesarios para el desarrollo de la misma.

Población. Participaron 14 panelistas con experiencia en diversos ámbitos de la macro, meso y microgestión sanitaria, investigación en servicios de salud y políticas farmacéuticas. Los participantes, cuyos nombres y cargos en el momento de la reunión se recogen en el anexo 1, fueron seleccionados por los autores (a propuesta de cualquiera de ellos) para componer un gru- 
po plural, con conocimientos, experiencias y trayectorias laborales en áreas muy diversas del sector sanitario (hospitales, atención primaria, administración sanitaria, gestión de centros a diversos niveles, clínica, docencia, investigación, calidad asistencial, cadena farmacéutica, evaluación e investigación evaluativa, salud pública y otros) y, en lo posible, con trayectorias mixtas en diversos puestos y áreas de las organizaciones sanitarias. La selección final se realizó por consenso de los autores con criterios subjetivos basados en su propio conocimiento del sector y no por un criterio formal explícito (currícula, publicaciones o similares).

Métodos. Se combinaron aspectos de tres técnicas grupales intentando estimular la creatividad del grupo para generar el máximo número de propuestas, desarrollar una reunión muy formalizada para mantener al grupo centrado en el objetivo cumpliendo los plazos de tiempo previstos y usar técnicas de priorización para ordenar la relevancia de las propuestas. Del brainstorming se adoptó la fase de reflexión silenciosa previa (que comparte con el grupo nominal), la imposibilidad de rechazar propuestas y el control de las interacciones negativas entre los participantes. De la técnica del grupo nominal se adoptaron las fases iniciales (confección de la pregunta, fase nominal, ronda de respuestas y conducción de la reunión) pero minimizando la ronda de discusión previa a las votaciones. De la técnica Rand se incorporó su sistema de valoración de escenarios (de 1 a 9, con la valoración final en 3 tramos: 1-3, 4-6 y 7-9) adaptado a la valoración de propuestas.

Pregunta. La pregunta a formular al grupo de panelistas fue preparada por los coordinadores del panel y se entregó por escrito a cada miembro del grupo previamente a la fase de reflexión silenciosa, acompañada de un contexto para delimitar la concreción, alcance y formato de las respuestas (anexo 2).
Dinámica de la reunión. Dado que el objetivo del grupo era identificar potenciales obstáculos y limitaciones así como valorar su importancia relativa, la dinámica del mismo se centro en la generación de ideas y en su posterior valoración/priorización, pero no en la discusión/argumentación de las mismas. Tras la presentación de los objetivos de la reunión y una breve explicación sobre la dinámica a seguir, el moderador planteó la pregunta junto a un contexto para delimitar el tipo de respuestas (anexo 2) a partir de la que cada miembro del grupo debía escribir, individualmente sin comunicarse con los demás panelistas, tantas propuestas como le fuera posible durante un plazo de 10 minutos. Una vez finalizado, se dio comienzo a la lectura de las respuestas de los participantes (por turno, una cada vez) mientras el moderador, que también actuó como panelista, las proyectaba para que estuvieran a la vista de todos. Aunque se permitió la aclaración del contenido de las propuestas, no se rechazó ninguna ni se permitió la discusión de las mismas o la confrontación de ideas. Al finalizar la ronda de respuestas se pidió a los panelistas que puntuaran, en silencio e individualmente, cada una de las respuestas. El rango de puntuaciones posible iba desde 1 (obstáculo o limitación mínimo) hasta 9 (obstáculo o limitación insalvable). Se remarcó la conveniencia de separar la valoración de los ítems de las personas que los habían propuesto y de no evitar las puntuaciones extremas.

Análisis. Descripción narrativa de las respuestas y su puntuación de prioridad. Para la descripción se utilizó la mediana, los percentiles $25\left(\mathrm{P}_{25}\right)$ y $75\left(\mathrm{P}_{75}\right)$ y la razón de variación entre el $\mathrm{P}_{75}$ y el $\mathrm{P}_{25}\left(\mathrm{RV}_{25-75}\right)$ que ofrece un parámetro de la dispersión de las puntuaciones. Conforme a la estructura de los rangos de puntuación, se había previsto una clasificación en obstáculos importantes (puntuaciones entre 7 y 9), de importancia media (puntuaciones entre 4 y 6 ) y poco importantes (puntuaciones entre 1 y 3 ) aunque, dada la experiencia y conocimientos 
del grupo, no se esperaba un elevado número de respuestas en el tramo de poca importancia. Las respuestas se clasificaron en 4 áreas temáticas desarrolladas ex-post por los coordinadores para facilitar su relato. Para ello se partió del ítem con mayor puntuación, se definió su área temática y se le añadieron los ítem relacionados. A continuación se realizó el mismo proceso con el siguiente ítem no incluido en el área previa, y así hasta agotar todos los ítem propuestos por los panelistas.

\section{RESULTADOS}

El panel propuso 77 ítems como posibles dificultades, obstáculos o limitaciones para el establecimiento de un "ente" oficial e independiente con capacidad para informar sobre la financiación y desinversión selectiva de las prestaciones del SNS. En el anexo 3 se recogen todas las propuestas numeradas de la 1 a la 77 según el orden de importancia (mediana y media) otorgado por la puntuación de los panelistas. Un total de 46 propuestas obtuvieron medianas de puntuación iguales o superiores a 7 puntos (obstáculos importantes) de las que 12 tuvieron medianas iguales o superiores a 8 puntos. El resto de propuestas se situó en la zona de importancia moderada (con puntuaciones inferiores a 7 pero superiores a 4), excepto 2 propuestas que obtuvieron medianas en el rango de 1-3 puntos.

La primera de las áreas temáticas en la que se clasificaron las propuestas de los panelistas reunió los ítem relativos a la voluntad política de los gobiernos estatal y autonómicos para introducir el CEA en el SNS y, en estrecha relación, los concernientes a la independencia y transparencia de los procesos de evaluación (tabla 1). Estas propuestas recogieron, en algunos casos de forma combinada en el mismo ítem, los obstáculos identificados por los panelistas en relación a las reticencias de gobiernos y personas que toman las decisiones a nivel sanitario respecto a la evaluación enfocada a la incorporación/desinversión de prestaciones sanitarias, la falta de confianza en la independencia y el buen gobierno del ente evaluador en un contexto de importantes conflictos de intereses y alta intromisión política, la tradición de falta de transparencia en la administración española, la ausencia de mecanismos para implicar a la ciudadanía o a los pacientes en los procesos evaluativos, y los conflictos de intereses derivados de la cercanía de este tipo de entes a la administración sanitaria. Los ítem propuestos en este apartado obtuvieron las mayores puntuaciones de los panelistas, copando 10 de los 15 primeros puestos según la valoración global de los panelistas (y 9 de las 12 valoraciones que alcanzaron medianas de 8 puntos).

La segunda área temática (tabla 2) incluyó los ítem relacionados con la estructura organizativa y la cultura del ente evaluador. Plantearon esencialmente la tensión entre un sistema sanitario descentralizado, en el que las CCAA han ido desarrollando sus propios mecanismos de evaluación de tecnologías y medicamentos, y la eficiencia de la actividad evaluadora que, por un lado, tiene que dar un servicio común al conjunto del SNS y, por otro, es una actividad con importantes economías de escala (incluso a nivel internacional). Tres de estos ítem se situaron entre los 15 primeros en importancia.

Una tercera área temática recogió aspectos relacionados con los procesos evaluativos y la capacidad de los resultados de evaluación para influir en la toma de decisiones (tabla 3), incluyendo la necesidad de cambios organizativos (y las posibles resistencias) para la implantación de algunas decisiones de desinversión, la ambigüedad de la relación entre la evidencia y la toma de decisiones concretas, una posible falta de legitimidad de las decisiones de desinversión cuando no se acompañan de políticas generales para reducir el despilfarro, la orientación de las agencias de evaluación hacia la valoración de la eficacia (frente a la eficiencia), la innovación (frente a las tecnologías establecidas) y los dispositivos (frente a los medicamentos), así como los propios límites de capacidad para evaluar un gran núme- 


\section{Tabla 1}

Obstáculos más importantes (mediana $\geq 7$ ) para establecer un "ente" evaluador de las prestaciones a incorporar o desincorporar de la cartera de servicios del SNS. Ítem relacionados con la voluntad política y la independencia y transparencia del ente

\begin{tabular}{|c|c|c|c|c|c|}
\hline Núm. & Descripción abreviada de los ítem. & Med. & $\mathrm{P}_{25}$ & $\mathrm{P}_{75}$ & $\mathrm{RV}$ \\
\hline 1 & $\begin{array}{l}\text { Escasa predisposición de los gobiernos y decisores para superar los conflictos de } \\
\text { intereses de la financiación selectiva con una agencia independiente y criterios } \\
\text { transparentes y vinculantes. }\end{array}$ & 8 & 7,3 & 9 & 1,2 \\
\hline 2 & No puede hablarse de eficiencia sin transparencia. & 8 & 7,3 & 9 & 1,2 \\
\hline 3 & Completa falta de voluntad política por parte del Gobierno estatal. & 8 & 6 & 9 & 1,5 \\
\hline 4 & $\begin{array}{l}\text { Falta definir una metodología clara y transparente que incluya la participación de } \\
\text { todos los agentes implicados. }\end{array}$ & 8 & 7 & 8,8 & 1,3 \\
\hline 6 & $\begin{array}{l}\text { Escasa o nula tradición jurídica (central y autonómica) en la creación de organis- } \\
\text { mos auténticamente independientes. }\end{array}$ & 8 & 6,3 & 8,8 & 1,4 \\
\hline 7 & $\begin{array}{l}\text { Falta de confianza en la independencia del ente: necesidad de generar transpa- } \\
\text { rencia en el origen de la financiación, en su gestión y en sus resultados. }\end{array}$ & 8 & 7 & 8 & 1,1 \\
\hline 8 & $\begin{array}{l}\text { Insuficiente independencia en la toma de decisiones (políticos: marco; conteni- } \\
\text { dos: gestores y operadores). }\end{array}$ & 8 & 7 & 8 & 1,1 \\
\hline 9 & $\begin{array}{l}\text { Escasa cultura evaluativa en los más altos niveles políticos (relacionada con la } \\
\text { escasa tradición de rendición de cuentas). }\end{array}$ & 8 & 7 & 8 & 1,1 \\
\hline 10 & $\begin{array}{l}\text { Poca tradición en la rendición de cuentas (en parte por la escasa implicación de } \\
\text { los ciudadanos). }\end{array}$ & 8 & 7 & 8 & 1,1 \\
\hline 15 & $\begin{array}{l}\text { Desconfianza ante la gobernanza del ente: criterios de selección de dirección y } \\
\text { personal. }\end{array}$ & 7,5 & 6,25 & 8 & 1,3 \\
\hline 16 & $\begin{array}{l}\text { Escasa predisposición política y gestora a tomar decisiones coherentes con las re- } \\
\text { comendaciones y a explicar el coste de oportunidad de no hacer nada. }\end{array}$ & 7,5 & 6 & 8 & 1,3 \\
\hline 26 & Falta de visión global/perspectiva del sector sanitario en el conjunto del gasto social. & 7 & 6,25 & 8 & 1,3 \\
\hline 28 & Ausencia de mecanismos para implicar a la ciudadanía en la toma de decisiones. & 7 & 6 & 8 & 1,3 \\
\hline 31 & $\begin{array}{l}\text { Falta transparencia en la financiación de los expertos que finalmente interpreta- } \\
\text { rán la evidencia. }\end{array}$ & 7 & 6 & 8 & 1,3 \\
\hline 35 & $\begin{array}{l}\text { Existencia de terrenos vedados a la evaluación (ej.: efectividad y eficiencia de } \\
\text { cambios organizativos como las concesiones y la gestión privada del asegura- } \\
\text { miento público). }\end{array}$ & 7 & 5,25 & 8 & 1,5 \\
\hline 36 & Demasiadas leyes y escasa voluntad de rectoría. & 7 & 5,25 & 8 & 1,5 \\
\hline 37 & $\begin{array}{l}\text { El organismo debería tener una perspectiva amplia (no sólo relacionada con la } \\
\text { cartera de servicios), y dependencia parlamentaria. }\end{array}$ & 7 & 5 & 8 & 1,6 \\
\hline 41 & Falta de tradición de involucrar al paciente en el proceso evaluativo. & 7 & 6,25 & 7,75 & 1,2 \\
\hline 44 & $\begin{array}{l}\text { Estado de partidos ("partitocracia") que han acabado por controlar las institucio- } \\
\text { nes públicas con la legitimidad electoral. }\end{array}$ & 7 & 6 & 7 & 1,2 \\
\hline
\end{tabular}

SNS: Sistema Nacional de Salud. Med: Mediana. P: Percentil. RV: razón de variación entre el $\mathrm{P}_{75}$ y el $\mathrm{P}_{25}$.

ro de prestaciones. Siete de los ítem de este apartado obtuvieron valoraciones con mediana $\geq 7$.
Finalmente, un cuarto epígrafe recogió los obstáculos relacionados con la percepción social y profesional de la evaluación de tecnologías (tabla 4), incluyendo aspectos 


\section{Tabla 2}

Obstáculos más importantes (mediana $\geq 7$ ) para establecer un "ente" evaluador de las prestaciones a incorporar o desincorporar de la cartera de servicios del SNS. Ítem relacionados con la estructura organizativa y la cultura del ente evaluador

\begin{tabular}{|c|c|c|c|c|c|}
\hline Núm. & Descripción abreviada de los ítem. & Med. & $\mathrm{P}_{25}$ & $\mathrm{P}_{75}$ & RV \\
\hline 5 & $\begin{array}{l}\text { La arquitectura del sistema sanitario español no favorece la existencia de un ente } \\
\text { evaluador único. }\end{array}$ & 8 & 7 & 8,8 & 1,3 \\
\hline 12 & Escasa capacidad de compartir las mejoras prácticas a nivel nacional e internacional. & 8 & 4,3 & 8 & 1,9 \\
\hline 13 & Excesivas duplicidades. No necesitamos “10” agencias de evaluación. & 7,5 & 6 & 8,8 & 1,5 \\
\hline 21 & Interés de las agencias autonómicas en mantener el status quo. & 7 & 5,25 & 8,75 & 1,7 \\
\hline 27 & $\begin{array}{l}\text { Incoherencia entre una agencia española centralizada y una sanidad descentralizada } \\
\text { y financiada por las Comunidades Autónomas. }\end{array}$ & 7 & 6,25 & 8 & 1,3 \\
\hline 43 & $\begin{array}{l}\text { Escasa asociación entre la cultura de evaluación con la innovación y formulas de } \\
\text { riesgo compartido. }\end{array}$ & 7 & 4,5 & 7,75 & 1,7 \\
\hline 46 & $\begin{array}{l}\text { Ausencia de un modelo organizativo de la agencias/agencias (o ente/s): no financia- } \\
\text { ción estable de grupos solventes con directrices claras. }\end{array}$ & 7 & 5 & 7 & 1,4 \\
\hline
\end{tabular}

SNS: Sistema Nacional de Salud; Med: Mediana: P: Percentil; RV: razón de variación entre el $\mathrm{P}_{75}$ y el $\mathrm{P}_{25}$.

\section{Tabla 3}

Obstáculos más importantes (mediana $\geq 7$ ) para establecer un "ente" evaluador de las prestaciones a incorporar o desincorporar de la cartera de servicios del SNS. Ítem relacionados con los procesos y resultados del ente evaluador

\begin{tabular}{|c|c|c|c|c|c|}
\hline Núm. & Descripción abreviada de los ítem. & Med. & $\mathrm{P}_{25}$ & $\mathrm{P}_{75}$ & RV \\
\hline 11 & $\begin{array}{l}\text { Las decisiones de desinversión no pueden separarse de cambios organizati- } \\
\text { vos (resistencias). }\end{array}$ & 8 & 6,3 & 8 & 1,3 \\
\hline 14 & Poco impacto de la evidencia en las decisiones políticas de salud (en general). & 7,5 & 7 & 8 & 1,1 \\
\hline 17 & Ambigüedad inferencial de la evaluación de tecnologías. & 7,5 & 6 & 8 & 1,3 \\
\hline 18 & $\begin{array}{l}\text { Baja robustez/aceptación metodológica: tiempo de respuesta excesivo, medida } \\
\text { de beneficios de baja validez y métodos de evaluación poco estandarizados. }\end{array}$ & 7,5 & 6 & 8 & 1,3 \\
\hline 19 & Mayor tradición en evaluar innovación que en evaluar desinversión. & 7,5 & 5,3 & 8 & 1,5 \\
\hline 22 & $\begin{array}{l}\text { Ausencia de discusión abierta e informada sobre las listas de "do not to do" } \\
\text { del NICE y otras organizaciones }\end{array}$ & 7 & 4,25 & 8,75 & 2,1 \\
\hline 23 & $\begin{array}{l}\text { La información necesaria para decidir suele no estar disponible en el momento } \\
\text { en que se requiere tomar la decisión (problemática de evaluación de la innova- } \\
\text { ción). }\end{array}$ & 7 & 7 & 8 & 1,1 \\
\hline 24 & $\begin{array}{l}\text { Falta de legitimidad de la desinversión si no se aplica también en otras polí- } \\
\text { ticas ("tocho", fraude fiscal, Bankia, y similares) }\end{array}$ & 7 & 7 & 8 & 1,1 \\
\hline 33 & $\begin{array}{l}\text { El "tempo" de la evaluación de tecnologías es diferente al tiempo de la toma de } \\
\text { decisiones. La evaluación requiere tiempo para desarrollar estudios válidos. }\end{array}$ & 7 & 5,25 & 8 & 1,5 \\
\hline 38 & $\begin{array}{l}\text { Evaluación muy centrada en la eficacia y menos en otros aspectos (eficien- } \\
\text { cia). }\end{array}$ & 7 & 4,5 & 8 & 1,8 \\
\hline 39 & $\begin{array}{l}\text { Extender la evaluación no sólo al qué sino también al cómo (práctica habi- } \\
\text { tual para disminuir la variabilidad clínica). }\end{array}$ & 7 & 4,25 & 8 & 1,9 \\
\hline
\end{tabular}

SNS: Sistema Nacional de Salud; Med: Mediana: P: Percentil; RV: razón de variación entre el $\mathrm{P}_{75}$ y el $\mathrm{P}_{25}$ NICE: National Institutes of Clinical Excellence. Listado completo de ítem en el anexo 1. 
Tabla 4

Obstáculos más importantes (mediana $\geq 7$ ) para establecer un "ente" evaluador de las prestaciones a incorporar o desincorporar de la cartera de servicios del SNS. Ítem relacionados con la percepción social y profesional de la evaluación de tecnologías

\begin{tabular}{|c|c|c|c|c|c|}
\hline Núm. & Descripción abreviada de los ítem. & Med. & $\mathrm{P}_{25}$ & $\mathrm{P}_{75}$ & RV \\
\hline 20 & $\begin{array}{l}\text { No saber gestionar la implicación de los profesionales sanitarios incorpo- } \\
\text { rando el concepto de eficiencia clínica en los procesos de evaluación. }\end{array}$ & 7 & 6 & 8,75 & 1,5 \\
\hline 25 & $\begin{array}{l}\text { Rechazo social a la desinversión. Falta de información al ciudadano del } \\
\text { verdadero coste-efectividad. }\end{array}$ & 7 & 6,25 & 8 & 1,3 \\
\hline 29 & $\begin{array}{l}\text { Dificultad de que el mensaje se entienda por parte de la población (percep- } \\
\text { ción de más recortes). }\end{array}$ & 7 & 6 & 8 & 1,3 \\
\hline 30 & $\begin{array}{l}\text { Coyuntura sociopolítica actual: agencias como herramientas legitimadoras } \\
\text { de recortes. }\end{array}$ & 7 & 6 & 8 & 1,3 \\
\hline 32 & $\begin{array}{l}\text { Baja demanda de la sociedad civil y los profesionales del sistema hacia un } \\
\text { organismo de este tipo. }\end{array}$ & 7 & 6 & 8 & 1,3 \\
\hline 34 & $\begin{array}{l}\text { Rechazo por parte de los clínicos a los criterios de coste-efectividad, e in- } \\
\text { cluso a los de efectividad comparada. }\end{array}$ & 7 & 5,25 & 8 & 1,5 \\
\hline 40 & Necesidad de difundir y explicar bien el porqué del trabajo y los resultados. & 7 & 4 & 8 & 2,0 \\
\hline 42 & Dificultad para visualizar potenciales ahorros y ganancias en salud. & 7 & 6 & 7,75 & 1,3 \\
\hline 45 & $\begin{array}{l}\text { Ausencia de convencimiento de que necesitamos este órgano con prestigio } \\
\text { interno y externo. }\end{array}$ & 7 & 5 & 7 & 1,4 \\
\hline
\end{tabular}

SNS: Sistema Nacional de Salud; Med: Mediana: P: Percentil; RV: razón de variación entre el $\mathrm{P}_{75} \mathrm{y}_{\text {el }} \mathrm{P}_{25}$.

relacionados con la incomprensión y el rechazo social a la exclusión de prestaciones y la desinversión, el rechazo por parte de los profesionales sanitarios y de las asociaciones sectoriales de pacientes a la aplicación de criterios de eficiencia y a la baja demanda de la sociedad civil y de la propia comunidad sanitaria de un organismo evaluador. Once de los ítem de este apartado obtuvieron valoraciones con mediana $\geq 7$.

\section{DISCUSIÓN}

Pese a las claras tendencias en Europa y cierta efervescencia evaluadora en el entorno español, la principal barrera identificada por los panelistas para la introducción de estas metodologías en nuestro país fue, precisamente, la falta de compromiso de las autoridades sanitarias del SNS, en especial del Ministerio de Sanidad, con la adopción de este tipo de estrategias de mejora de la eficiencia del sistema sanitario. Esta escasa voluntad política se atribuye esencialmente a la falta de trasparencia, de cultura evaluadora y de interés en la creación de organismos independientes de la administración pública española, que vería limitada su actual discrecionalidad para la incorporación de medicamentos y la fijación de sus precios.

Una segunda barrera tendría que ver con las tensiones entre la actividad evaluadora, cuya homogeneización y centralización tiene importantes ventajas, y un sistema sanitario descentralizado que ha ido desarrollando periféricamente una importante actividad en el terreno de la evaluación de medicamentos y tecnologías y que las CCAA podrían querer mantener en el tiempo sin grandes cambios. La conjunción de ambos elementos (Gobierno central intentando mantener su capacidad discrecional sobre las decisiones de cartera de servicios y precios, y CCAA intentando mantener una fragmentada capacidad evaluadora) añadiría un componente de disputa de competencias y contribuiría de forma notable al mantenimiento del status quo actual. 
El resto de barreras identificadas por los panelistas son comunes a las relatadas en otros países y se relacionan con las dificultades generales de los métodos de evaluación económica para apoyar la toma de decisiones ${ }^{19-22}$, con la percepción, especialmente agudizada en el contexto actual, de que estos métodos se emplearán para dar un barniz científico a los "recortes" antes que para una valoración equilibrada de costes y beneficios, y con la oposición de las asociaciones de pacientes a las restricción de prestaciones a sus colectivos específicos ${ }^{23}$. También se apunta, y esto volvería a ser un aspecto específico del SNS, la limitada capacidad investigadora/evaluadora del SNS, basada en equipos de evaluación pequeños, fragmentados $\mathrm{y}$, en buena parte, con estructuras organizativas muy débiles y periféricas en sus propias organizaciones, con grandes dificultades para incorporar o retener personal investigador bien formado o para abordar un elevado número de evaluaciones.

Aunque existe literatura sobre el impacto de la evaluación sobre las decisiones sanitarias $^{24}$, sólo hemos encontrado una publicación -una revisión narrativa- que intente sistematizar las barreras y limitaciones para la introducción de este tipo de análisis en la toma de decisiones sanitarias. Aunque la revisión se centra en un entorno -países asiáticos- muy diferente al contexto del SNS español ${ }^{25}$, algunas de las barreras identificadas son coincidentes con las señaladas por los panelistas en este estudio (relacionadas con la capacidad investigadora, desconfianza desde la política, ciudadanos y profesionales, temores sobre su uso para un racionamiento exagerado y alguna otra). Sin embargo, mientas el estudio asiático hace hincapié en las barreras de falta de conocimiento entre los potenciales usuarios y en la formación de los decisores, este un aspecto escasamente mencionado en nuestro estudio, con sólo referencias en 3 ítem $(53,72$ y 74) que obtuvieron puntuaciones intermedias o bajas y que sugiere que los panelistas opinaban que esta no sería una barrera relevante en nuestro contexto.
Entre las limitaciones de este trabajo, además de las derivadas de la propia metodología utilizada que, obviamente, hace que los resultados respondan a las opiniones de los expertos, cabe señalar, en primer lugar, las vinculadas a la selección de los panelistas, que no representan -ni se pretendía- a los agentes del sistema sanitario. El objetivo del grupo era esencialmente identificar obstáculos, antes que negociar las posibles ideas entre los grupos de interés que conviven en el SNS y su entorno. Por ello, los panelistas se seleccionaron entre personas que tenían un buen conocimiento del SNS desde diversas perspectivas gestoras, analíticas o asistenciales, pero intentando evitar que fueran "representantes" de colectivos concretos. En este mismo sentido, el panel -en buena parte por aspectos prácticos de minimización de costes de desplazamiento- tenía un claro predominio de participantes de Cataluña $y$, aunque se intentó mantener una visión general, algunas de las respuestas pueden tener relación con perspectivas específicas o de especial relevancia en esa comunidad autónoma. En segundo lugar, la forma de clasificar los ítem bajo diferentes epígrafes es también subjetiva, siendo posible que otras interpretaciones dieran lugar a agrupaciones diferentes, máxime cuando algunos ítem son lo suficientemente genéricos como para ofrecer diversas posiblidades de clasificación.

La evaluación económica de medicamentos y tecnologías está alcanzando su edad dorada en Europa ${ }^{26}$. La principal causa de este florecimiento es, sin duda, el impulso de las autoridades públicas que utilizan esta metodología como un instrumento esencial para la adopción, fijación de precios y difusión de las innovaciones sanitarias, en un esfuerzo por combinar el acceso de los ciudadanos a los avances terapéuticos con la sostenibilidad financiera de los sistemas sanitarios públicos, que ven amenazada por la adopción indiscriminada de nuevos y caros medicamentos y dispositivos. En el SNS existe, al menos desde la Ley del 
Medicamento de $1990^{28}$, un marco normativo que ofrece amparo legal a la utilización de criterios de eficiencia para la financiación selectiva de medicamentos, extendido a otras prestaciones por el Real Decreto $1030 / 2006^{29}$ por el que se establece la cartera de servicios comunes del SNS y revalidado por el Real Decreto-ley 16/2012, de 20 de abril 27 que, además de citar expresamente los criterios de "eficiencia" y de "impacto económico y organizativo", recoge expresamente la participación en estas evaluaciones de la "Red Española de Agencias de Evaluación de Tecnologías Sanitarias y Prestaciones".

También existen diversas agencias de evaluación de tecnologías (la dependiente del Ministerio de Sanidad a través del Instituto de Salud Carlos III y las agencias dependientes de diversas Comunidades Autónomas) $)^{30}$ o los centros de evaluación de medicamentos de las CCAA (que incluso iniciaron una cierta coordinación a través del Comité Mixto de Evaluación de Nuevos Medicamentos en el que participaban Andalucía, País Vasco, Navarra, Aragón y Cataluña). Adicionalmente existe una larga tradición de estudios de evaluación económi$\mathrm{ca}^{31,32}$, sociedades profesionales y expertos que reclaman el desarrollo de políticas explícitas para la incorporación de tecnologías sanitarias ${ }^{33}$, se han avanzado propuestas de estandarización metodológica ${ }^{34,35} \mathrm{y}$, al menos en algunas áreas, como los medicamentos hospitalarios, las sociedades profesionales han desarrollado un notable esfuerzo de coordinación para hacer más eficiente la evaluación de nuevos fármacos (especialmente el Grupo GENESIS de la Sociedad Española de Farmacia Hospitalaria), que en muchos casos incluyen información sobre costes, coste-efectividad e impacto presupuestario ${ }^{36}$, aunque muy raramente una evaluación económica o de impacto presupuestario formal.

Evaluar medicamentos, tecnologías y otras prestaciones para informar su incorpo- ración (o su desinversión) a la cartera de servicios del SNS es uno de los instrumentos más potentes con que cuenta un sistema sanitario para garantizar su sostenibilidad y, sobre todo, para priorizar sus recursos en aras de conseguir el mayor bienestar colectivo posible. Esta actividad no se limita a la evaluación económica y a la del impacto presupuestario, pero también incluye estas modalidades de evaluación que, hasta la fecha, han sido eludidas por el SNS. En España existe actualmente una importante capacidad evaluadora de tecnologías y medicamentos que, no obstante, se halla fragmentada entre diversas organizaciones y entre diversas CCAA, limitando ostensiblemente su potencial para la mejora del sistema de atención. Nuestro trabajo, aunque identifica problemas de diverso tipo y nivel, sitúa en el plano más político del sistema sanitario las principales dificultades para avanzar en este terreno. Un aspecto importante porque probablemente no incorporar la evaluación económica a la toma de decisiones es un lujo que el Sistema Nacional de Salud no puede permitirse ${ }^{26}$.

Aunque existen numerosos y variados obstáculos para desarrollar la capacidad del SNS en incorporar o excluir prestaciones de la cartera de servicios en función de la evaluación de su efectividad y eficiencia, los expertos sitúan en el plano más político del sistema sanitario (voluntad política, transparencia, gobernanza) las principales dificultades para avanzar en este terreno.

\section{BIBLIOGRAFÍA}

1. Clement FM, Harris A, Li JJ, Yong K, Lee KM, Manns BJ. Using effectiveness and cost-effectiveness to make drug coverage decisions: a comparison of Britain, Australia, and Canada. JAMA. 2009;302(13):1437-43.

2. Oliva J, Antoñanzas F, Rivero-Arias O. Evaluación económica y toma de decisiones en salud. El papel de la evaluación económica en la adopción y la difusión de tecnologías sanitarias. Informe SESPAS 2008. Gac Sanit. 2008;22 Suppl 1:137-42. 
3. Noorani HZ, Husereau DR, Boudreau R, Skidmore B. Priority setting for health technology assessments: a systematic review of current practical approaches. Int J Technol Assess Health Care. 2007;23(3):310-5.

4. Hutton J, McGrath C, Frybourg JM, Tremblay M, Bramley-Harker E, Henshall C. Framework for describing and classifying decision-making systems using technology assessment to determine the reimbursement of health technologies (fourth hurdle systems). Int J Technol Assess Health Care. 2006;22(1):10-8.

5. Morgan SG, McMahon M, Mitton C, Roughead E, Kirk R, Kanavos P, Menon D. Centralized drug review processes in Australia, Canada, New Zealand, and the United kingdom. Health Aff (Millwood). 2006;25(2):337-47.

6. Williams I, McIver S, Moore D, Bryan S. The use of economic evaluations in NHS decision-making: a review and empirical investigation. Health Technol Assess. 2008;12(7):iii, ix-x, 1-175.

7. Anell A, Persson U. Reimbursement and clinical guidance for pharmaceuticals in Sweden: do health-economic evaluations support decision making? Eur J Health Econ. 2005;6(3):274-9.

8. Taylor RS, Drummond MF, Salkeld G, Sullivan SD. Inclusion of cost effectiveness in licensing requirements of new drugs: the fourth hurdle. BMJ. 2004;329(7472):972-5.

9. Campillo-Artero C, Bernal-Delgado E. Disinvestment in health: fundamentals, clarifications, experiences and perspectives. Gac Sanit. 2012; 27(2):175-9.

10. Corbacho B, Pinto-Prades JL. Health economic decision-making: a comparison between UK and Spain. Br Med Bull. 2012;103(1):5-20.

11. Cappellaro G, Fattore G, Torbica A. Funding health technologies in decentralized systems: A comparison between Italy and Spain. Health Policy. 2009;92(23):313-21.

12. Sánchez F I, Martín D. Revisión de medicamentos en España: Prosereme y nuevos enfoques. Farm Hosp. 1994; 18:162-5.

13. Boletín Oficial del Estado. Resolución de 2 de agosto de 2012, de la Dirección General de Cartera Básica de Servicios del Sistema Nacional de Salud y Farmacia, por la que se procede a la actualización de la lista de medicamentos que quedan excluidos de la prestación farmacéutica en el Sistema Nacional de Salud. BOE núm. 197 de 17/8/2012.
14. Delbecq AL, Van de Ven A, Gustafson DH. Técnicas grupales para la planeación. México: Ed. Trillas; 1984.

15. Van de Ven AH, Delbecq AL. The Nominal Group as a Research Instrument for Exploratory Health Studies. Am J Publ Health. 1972; 62:337-42.

16. Peiró S, Portella E. El grupo nominal en el entorno sanitario. Valencia: Institut Valencià d'Estudis en Salut Pública; 1993.

17. Fink A, Kosecoff J, Chassin M, Brook RH. Consensus Methods: Characteristics and Guidelines for Use. Am J Public Health. 1984; 74:979-83.

18. Peiró S, Portella E. No todo es acuerdo en el consenso. Limitaciones de los métodos de consenso en los servicios de salud. Gac Sanit. 1993;7:294300 .

19. López Bastida J, Oliva J, Antoñanzas F, GarcíaAltés A, Gisbert R, Mar J, Puig-Junoy J. [A proposed guideline for economic evaluation of health technologies]. Gac Sanit. 2010;24(2):154-70.

20. Oliva J, Puig-Junoy J, Bernal E. Evaluacion economica de medicamentos: experiencias y vias de avance. Gac Sanit. 2008; 22(4):358-61.

21. Neumann PJ, Johannesson M. From principle to public policy: using cost-effectiveness analysis. Health Aff (Millwood). 1994;13(3):206-14.

22. Raftery J. Methodological limitations of costeffectiveness analysis in health care: implications for decision making and service provision. J Eval Clin Pract. 1999;5(4):361-6.

23. Drummond M, Sculpher M. Common methodological flaws in economic evaluations. Med Care. 2005;43(7 Suppl):5-14.

24. Drummond M. Twenty Years of Using Economic Evaluations for Reimbursement Decisions. What Have We Achieved? York: Centre for Health Economics. University of York; 2012.

25. Yothasamut J, Tantivess S, Teerawattananon Y. Using economic evaluation in policy decisionmaking in Asian countries: mission impossible or mission probable? Value Health. 2009;12 Suppl 3:S26-30.

26. Puig-Junoy J, Oliva J. Evaluación económica de intervenciones sanitarias: el coste de oportunidad de no evaluar. Reumatol Clin. 2009;5(6):241-3. 
27. Boletín Oficial del Estado. Real Decreto-ley (RDL) 16/2012, de 20 de abril, de medidas urgentes para garantizar la sostenibilidad del Sistema Nacional de Salud y mejorar la calidad y seguridad de sus prestaciones. BOE núm. 98, de 24 de abril de 2012 (correcciones en BOE núm 116, de 15 de mayo de 2012).

28. Boletín Oficial del Estado. Ley 25/1990, de 20 de diciembre, del Medicamento. BOE núm 306 de 22/12/1990.

29. Boletín Oficial del Estado. Real Decreto $1030 / 2006$, de 15 de septiembre, por el que se establece la cartera de servicios comunes del Sistema Nacional de Salud y el procedimiento para su actualización. BOE núm 222 de 16/9/2006.

30. Sampietro-Colom L, Asua J, Briones E, Gol J; AuNETS Group. History of health technology assessment: Spain. Int J Technol Assess Health Care. 2009;25 Suppl 1:163-73.

31. Catalá-López F, García-Altés A. Evaluación económica de intervenciones sanitarias en España durante el periodo 1983-2008. Rev Esp Salud Publica. 2010;84(4):353-69.

32. Catalá-López F, Alonso-Arroyo A, AleixandreBenavent R, Ridao M, Bolaños M, García-Altés A, Sanfélix-Gimeno G, Peiró S. Coauthorship and institutional collaborations on cost-effectiveness analyses: a systematic network analysis. PLoS One. 2012;7(5):e38012.

33. Asociación de Economía de la Salud. Posición de la Asociación de Economía de la Salud en relación a la necesidad de un mayor uso de la evaluación económica en las decisiones que afectan a la financiación pública de las prestaciones y tecnologías en el Sistema Nacional de Salud. Barcelona, Marzo 2008 . [Disponible en: www.aes.es/Publicaciones/AESEE2.pdf; acceso en 18 agosto, 2013].

34. López-Bastida J, Oliva J, Antoñanzas F, GarcíaAltés A, Gisbert R, Mar J, Puig-Junoy J. Spanish recommendations on economic evaluation of health t e chnologies. Eur J Health Econ. 2010;11(5):513-20.

35. Donaldson C, Currie G, Mitton C. Cost effectiveness analysis in health care: contraindications. BMJ. 2002;325(7369):891-4.

36. Devlin N, Appleby J, Parkin D. Patients' views of explicit rationing: what are the implications for health service decision-making? J Health Serv Res Policy. 2003;8(3):183-6. 


\section{Anexo 1}

\section{Participantes en el panel de expertos (orden alfabético)}

\begin{tabular}{|c|c|c|c|}
\hline Nombre & Posición laboral & Profesión & Localidad \\
\hline Olinda Ania & $\begin{array}{l}\text { Subdirectora del Institut d'Estudis de la } \\
\text { Salut, Catsalut. }\end{array}$ & Médica & Barcelona \\
\hline Josep María Argimón & $\begin{array}{l}\text { Gerente de Compra y Evaluación de Ser- } \\
\text { vicios, Catsalut. }\end{array}$ & Médico & Barcelona \\
\hline Joan J. Artells i Herrero & Analista de política sanitaria & Economista & Barcelona \\
\hline Xavier Castells Oliveres & $\begin{array}{l}\text { Jefe del Servicio de Epidemiología y } \\
\text { Evaluación, Hospital del Mar-Parc de } \\
\text { Salut Mar. }\end{array}$ & Médico & Barcelona \\
\hline Jordi Colomer i Mascaró & $\begin{array}{l}\text { Facultativo Especialista de Cirugía Ge- } \\
\text { neral, Hospital de Viladecans. }\end{array}$ & Médico & $\begin{array}{l}\text { Viladecans, } \\
\text { Barcelona }\end{array}$ \\
\hline Joan Escarrabill & $\begin{array}{l}\text { Director de la Unidad de Liderazgo e In- } \\
\text { novación, AIAQS, Catsalut. }\end{array}$ & Médico & Barcelona \\
\hline Roser Fernández & $\begin{array}{l}\text { Secretaria General del Departamento de } \\
\text { Salud de la Generalitat de Catalunya. }\end{array}$ & Médica & Barcelona \\
\hline Anna García Altés & $\begin{array}{l}\text { Directora del Observatorio de Política } \\
\text { Sanitaria, AIAQS, Catsalut. }\end{array}$ & Economista & Barcelona \\
\hline Joan Gené Badia & $\begin{array}{l}\text { Médico de Atención Primaria, Consorcio } \\
\text { de Atención Primaria de Salud del En- } \\
\text { sanche (CAPSE). }\end{array}$ & Médico & Barcelona \\
\hline Concha Marzo Carpio & $\begin{array}{l}\text { Directora de Market Acces, Novartis } \\
\text { Farmacéutica. }\end{array}$ & Farmacéutica & Barcelona \\
\hline Enrique Molina Pérez de los Cobos & Estudiante de Grado de Medicina & Estudiante & Albacete \\
\hline Juan Oliva Moreno & $\begin{array}{l}\text { Profesor Titular de la Universidad de } \\
\text { Castilla la Mancha }\end{array}$ & Economista & Toledo \\
\hline Salvador Peiró * & $\begin{array}{l}\text { Jefe del área de investigación en servi- } \\
\text { cios de salud, Centro Superior de Inves- } \\
\text { tigación en Salud Pública (CSISP). }\end{array}$ & Médico & Valencia \\
\hline Joan Carles Serrat & CIC Control & Farmacéutico- Analista & Barcelona \\
\hline
\end{tabular}

\section{Anexo 2}

\section{Pregunta efectuada a los expertos del panel}

¿Qué dificultades, limitaciones y obstáculos deberían sortearse para establecer un "ente” oficial e independiente con capacidad para informar (con criterios explícitos y métodos contrastados y rigurosos que tengan en cuenta la efectividad, seguridad, eficiencia e impacto presupuestario) sobre la financiación (y desinversión) selectiva de las prestaciones del Sistema Nacional de Salud?

- Formular las ideas en forma de "ítems" de una frase.

- Los "ítem" deben tener un nivel de concreción "adecuado": ni tan genéricos que carezcan de valor ni tan micro que sólo tengan sentido en un entorno muy definido.

- Los “ítem” pueden incluir un amplio abanico de aspectos: políticos, legales, reticencias, conflictos de intereses, limitaciones técnicas o metodológicas, organizativos, burocráticos, de costes, capacidad investigadora, ... 
Anexo 3

Listado completo de ítem propuestos por los panelistas, con las puntaciones media, mediana, percentiles 25 y 75 y razón de variación entre ellos.

\begin{tabular}{|c|c|c|c|c|c|c|}
\hline Núm. & Descripción abreviada de los ítem. & Media & Med & $\mathrm{P}_{25}$ & $\mathrm{P}_{75}$ & RV \\
\hline 1 & $\begin{array}{l}\text { Escasa predisposición de los gobiernos y decisores para superar los conflictos } \\
\text { de intereses de la financiación selectiva con una agencia independiente y crite- } \\
\text { rios transparentes y vinculantes. }\end{array}$ & 8,1 & 8 & 7,25 & 9 & 1,2 \\
\hline 2 & No puede hablarse de eficiencia sin transparencia. & 7,7 & 8 & 7,25 & 9 & 1,2 \\
\hline 3 & Completa falta de voluntad política por parte del Gobierno estatal. & 7,2 & 8 & 6 & 9 & 1,5 \\
\hline 4 & $\begin{array}{l}\text { Falta definir una metodología clara y transparente que incluya la participación } \\
\text { de todos los agentes implicados. }\end{array}$ & 7,4 & 8 & 7 & 8,75 & 1,3 \\
\hline 5 & $\begin{array}{l}\text { La arquitectura del sistema sanitario español no favorece la existencia de un } \\
\text { ente evaluador único. }\end{array}$ & 7,3 & 8 & 7 & 8,75 & 1,3 \\
\hline 6 & $\begin{array}{l}\text { Escasa o nula tradición jurídica (central y autonómica) en la creación de orga- } \\
\text { nismos auténticamente independientes. }\end{array}$ & 7,3 & 8 & 6,25 & 8,75 & 1,4 \\
\hline 7 & $\begin{array}{l}\text { Falta de confianza en la independencia del ente: necesidad de generar transpa- } \\
\text { rencia en el origen de la financiación, en su gestión y en sus resultados. }\end{array}$ & 7,8 & 8 & 7 & 8 & 1,1 \\
\hline 8 & $\begin{array}{l}\text { Insuficiente independencia en la toma de decisiones (políticos: marco; conteni- } \\
\text { dos: gestores y operadores). }\end{array}$ & 7,6 & 8 & 7 & 8 & 1,1 \\
\hline 9 & $\begin{array}{l}\text { Escasa cultura evaluativa en los más altos niveles políticos (relacionada con la } \\
\text { escasa tradición de rendición de cuentas). }\end{array}$ & 7,5 & 8 & 7 & 8 & 1,1 \\
\hline 10 & $\begin{array}{l}\text { Poca tradición en la rendición de cuentas (en parte por la escasa implicación de } \\
\text { los ciudadanos). }\end{array}$ & 7,2 & 8 & 7 & 8 & 1,1 \\
\hline 11 & $\begin{array}{l}\text { Las decisiones de desinversión no pueden separarse de cambios organizativos } \\
\text { (resistencias). }\end{array}$ & 7,3 & 8 & 6,25 & 8 & 1,3 \\
\hline 12 & $\begin{array}{l}\text { Escasa capacidad de compartir las mejoras prácticas a nivel nacional e interna- } \\
\text { cional. }\end{array}$ & 6,6 & 8 & 4,25 & 8 & 1,9 \\
\hline 13 & Excesivas duplicidades. No necesitamos "10" agencias de evaluación. & 6,9 & 7,5 & 6 & 8,75 & 1,5 \\
\hline 14 & Poco impacto de la evidencia en las decisiones políticas de salud (en general). & 7,3 & 7,5 & 7 & 8 & 1,1 \\
\hline 15 & $\begin{array}{l}\text { Desconfianza ante la gobernanza del ente: criterios de selección de dirección y } \\
\text { personal. }\end{array}$ & 6,8 & 7,5 & 6,25 & 8 & 1,3 \\
\hline 16 & $\begin{array}{l}\text { Escasa predisposición política y gestora a tomar decisiones coherentes con las } \\
\text { recomendaciones y a explicar el coste de oportunidad de no hacer nada. }\end{array}$ & 7,2 & 7,5 & 6 & 8 & 1,3 \\
\hline 17 & Ambigüedad inferencial de la evaluación de tecnologías. & 6,9 & 7,5 & 6 & 8 & 1,3 \\
\hline 18 & $\begin{array}{l}\text { Baja robustez/aceptación metodológica: tiempo de respuesta excesivo, medida } \\
\text { de beneficios de baja validez y método de evaluación poco estandarizado. }\end{array}$ & 6,9 & 7,5 & 6 & 8 & 1,3 \\
\hline 19 & Más tradición en evaluar innovación que en evaluar desinversión. & 6,7 & 7,5 & 5,25 & 8 & 1,5 \\
\hline 20 & $\begin{array}{l}\text { No saber gestionar la implicación de los profesionales sanitarios incorporando } \\
\text { el concepto de eficiencia clínica en los procesos de evaluación. }\end{array}$ & 6,9 & 7 & 6 & 8,75 & 1,5 \\
\hline 21 & Interés de las agencias autonómicas en mantener en el status quo. & 6,6 & 7 & 5,25 & 8,75 & 1,7 \\
\hline 22 & $\begin{array}{l}\text { Ausencia de discusión abierta e informada sobre las listas de "do not to do" del } \\
\text { NICE y otras organizaciones. }\end{array}$ & 6,6 & 7 & 4,25 & 8,75 & 2,1 \\
\hline 23 & $\begin{array}{l}\text { La información necesaria para decidir suele no estar disponible en el momento en } \\
\text { que se requiere tomar la decisión (problemática de evaluación de la innovación). }\end{array}$ & 7,1 & 7 & 7 & 8 & 1,1 \\
\hline 24 & $\begin{array}{l}\text { Falta de legitimidad de la desinversión si no se aplica también en otras políticas } \\
\text { ("tocho", fraude fiscal, Bankia, ...). }\end{array}$ & 6,9 & 7 & 7 & 8 & 1,1 \\
\hline
\end{tabular}




\section{Anexo 3 (continuación)}

\begin{tabular}{|c|c|c|c|c|c|c|}
\hline Núm. & Descripción abreviada de los ítem & Media & Med & $\mathrm{P}_{25}$ & $\mathrm{P}_{75}$ & RV \\
\hline 25 & $\begin{array}{l}\text { Rechazo social a la desinversión. Falta de información al ciudadano del ver- } \\
\text { dadero coste-efectividad. }\end{array}$ & 7,1 & 7 & 6,25 & 8 & 1,3 \\
\hline 26 & $\begin{array}{l}\text { Falta de visión global/perspectiva del sector sanitario en el conjunto del } \\
\text { gasto social. }\end{array}$ & 6,7 & 7 & 6,25 & 8 & 1,3 \\
\hline 27 & $\begin{array}{l}\text { Incoherencia entre una agencia española centralizada y una sanidad descen- } \\
\text { tralizada y financiada por las Comunidades Autónomas. }\end{array}$ & 6,6 & 7 & 6,25 & 8 & 1,3 \\
\hline 28 & $\begin{array}{l}\text { Ausencia de mecanismos para implicar a la ciudadanía en la toma de deci- } \\
\text { siones. }\end{array}$ & 6,9 & 7 & 6 & 8 & 1,3 \\
\hline 29 & $\begin{array}{l}\text { Dificultad de que el mensaje se entienda por parte de la población (percep- } \\
\text { ción de más recortes). }\end{array}$ & 6,9 & 7 & 6 & 8 & 1,3 \\
\hline 30 & $\begin{array}{l}\text { Coyuntura sociopolítica actual: agencias como herramientas legitimadoras } \\
\text { de recortes. }\end{array}$ & 6,8 & 7 & 6 & 8 & 1,3 \\
\hline 31 & $\begin{array}{l}\text { Falta transparencia en la financiación de los expertos que finalmente inter- } \\
\text { pretarán la evidencia. }\end{array}$ & 6,6 & 7 & 6 & 8 & 1,3 \\
\hline 32 & $\begin{array}{l}\text { Baja demanda de la sociedad civil y los profesionales del sistema hacia un } \\
\text { organismo de este tipo. }\end{array}$ & 6,6 & 7 & 6 & 8 & 1,3 \\
\hline 33 & $\begin{array}{l}\text { El "tempo" de la evaluación de tecnologías es diferente al tiempo de la to- } \\
\text { ma de decisiones. La evaluación requiere tiempo para desarrollar estudios } \\
\text { válidos. }\end{array}$ & 6,6 & 7 & 5,25 & 8 & 1,5 \\
\hline 34 & $\begin{array}{l}\text { Rechazo por parte de los clínicos a los criterios de coste-efectividad, e in- } \\
\text { cluso a los de efectividad comparada. }\end{array}$ & 6,6 & 7 & 5,25 & 8 & 1,5 \\
\hline 35 & $\begin{array}{l}\text { Existencia de terrenos vedados a la evaluación (ej.: efectividad y eficiencia } \\
\text { de cambios organizativos como las concesiones y la gestión privada del ase- } \\
\text { guramiento público). }\end{array}$ & 6,6 & 7 & 5,25 & 8 & 1,5 \\
\hline 36 & Demasiadas leyes y escasa voluntad de rectoría. & 6,6 & 7 & 5,25 & 8 & 1,5 \\
\hline 37 & $\begin{array}{l}\text { El organismo debería tener una perspectiva amplia (no sólo relacionada con } \\
\text { la cartera de servicios) y dependencia parlamentaria. }\end{array}$ & 6,5 & 7 & 5 & 8 & 1,6 \\
\hline 38 & $\begin{array}{l}\text { Evaluación muy centrada en la eficacia y menos en otros aspectos (eficien- } \\
\text { cia). }\end{array}$ & 6,5 & 7 & 4,5 & 8 & 1,8 \\
\hline 39 & $\begin{array}{l}\text { Extender la evaluación no sólo al qué sino también al cómo (práctica habi- } \\
\text { tual para disminuir la variabilidad clínica). }\end{array}$ & 6,1 & 7 & 4,25 & 8 & 1,9 \\
\hline 40 & Necesidad de difundir y explicar bien el porque del trabajo y los resultados. & 6,2 & 7 & 4 & 8 & 2,0 \\
\hline 41 & Falta de tradición de involucrar al paciente en el proceso evaluativo. & 6,8 & 7 & 6,25 & 7,75 & 1,2 \\
\hline 42 & Dificultad para visualizar potenciales ahorros y ganancias en salud. & 6,6 & 7 & 6 & 7,75 & 1,3 \\
\hline 43 & $\begin{array}{l}\text { Escasa asociación entre la cultura de evaluación con la innovación y for- } \\
\text { mulas de riesgo compartido. }\end{array}$ & 6,3 & 7 & 4,5 & 7,75 & 1,7 \\
\hline 44 & $\begin{array}{l}\text { Estado de partidos ("partitocracia") que han acabado por controlar las insti- } \\
\text { tuciones públicas con la legitimidad electoral. }\end{array}$ & 6,3 & 7 & 6 & 7 & 1,2 \\
\hline 45 & $\begin{array}{l}\text { Ausencia de convencimiento de que necesitamos este órgano con prestigio } \\
\text { interno y externo. }\end{array}$ & 6,4 & 7 & 5 & 7 & 1,4 \\
\hline 46 & $\begin{array}{l}\text { Ausencia de un modelo organizativo de la agencias/agencias (o ente/s): no } \\
\text { financiación estable de grupos solventes con directrices claras. }\end{array}$ & 6,1 & 7 & 5 & 7 & 1,4 \\
\hline 47 & $\begin{array}{l}\text { Escaso conocimiento de la eficiencia de los proveedores de análisis de la } \\
\text { eficiencia: desconocimiento del funcionamiento de las agencias de evalua- } \\
\text { ción, su relevancia e impacto. }\end{array}$ & 6,6 & 6,5 & 6 & 8 & 1,3 \\
\hline 48 & Acotar el limite de su independencia a la rendición de cuentas parlamentaria. & 6,3 & 6,5 & 5,25 & 8 & 1,5 \\
\hline 49 & ¿Por qué otra agencia? ¿Más burocracia? & 5,9 & 6,5 & 4 & 8 & 2,0 \\
\hline
\end{tabular}




\section{Anexo 3 (continuación)}

\begin{tabular}{|c|c|c|c|c|c|c|}
\hline Núm. & Descripción abreviada de los ítem & Media & Med & $\mathrm{P}_{25}$ & $\mathrm{P}_{75}$ & RV \\
\hline 50 & $\begin{array}{l}\text { Falta flexibilidad jurídica y burocrática para que la efectividad y eficiencia sea el argu- } \\
\text { mento principal en la toma de decisiones. }\end{array}$ & 6,4 & 6,5 & 6 & 7,75 & 1,3 \\
\hline 51 & No confundir la decisión experta con la decisión política. Separar limites. & 6,4 & 6,5 & 6 & 7,75 & 1,3 \\
\hline 52 & Dificultad para reclutar y retener expertos independientes y aglutinar conocimiento. & 5,9 & 6,5 & 4,5 & 7,75 & 1,7 \\
\hline 53 & $\begin{array}{l}\text { La estructura de la fuerza de trabajo médica (especialización) dificulta la readaptación (y } \\
\text { crea resistencias). }\end{array}$ & 6,1 & 6,5 & 5,25 & 7 & 1,3 \\
\hline 54 & $\begin{array}{l}\text { Dificultades para superar las contingencias competenciales y optimizar los recursos a ni- } \\
\text { vel nacional e internacional. }\end{array}$ & 6,1 & 6,5 & 5 & 7 & 1,4 \\
\hline 55 & $\begin{array}{l}\text { Dispersión de posiciones acerca del carácter consultivo, informativo o prescriptivo de la } \\
\text { evidencia para la toma decisiones. }\end{array}$ & 5,9 & 6,5 & 5 & 7 & 1,4 \\
\hline 56 & Más aceptación social del error tipo I que del error tipo II. & 6,3 & 6 & 6 & 8 & 1,3 \\
\hline 57 & $\begin{array}{l}\text { Incomodidad en la estructura actual del estado autonómico para determinadas Comuni- } \\
\text { dades Autónomas. }\end{array}$ & 6,0 & 6 & 4,5 & 7,75 & 1,7 \\
\hline 58 & Desconfianza hacia la labor de organismos independientes en el ámbito de la decisión. & 6,0 & 6 & 4,25 & 7,75 & 1,8 \\
\hline 59 & $\begin{array}{l}\text { Algunas decisiones tienen aspectos enormemente políticos más allá de las evidencias } \\
\text { (ej.: aborto). }\end{array}$ & 5,9 & 6 & 4,25 & 7,75 & 1,8 \\
\hline 60 & Falta de liderazgo y coordinación a nivel central (agencias por libre). & 5,9 & 6 & 4,25 & 7,75 & 1,8 \\
\hline 61 & $\begin{array}{l}\text { Incapacidad del sector para aglutinar de forma ponderada a los grupos que operan en to- } \\
\text { dos los ámbitos del sistema (desde la planificación a la compra-provisión). }\end{array}$ & 5,8 & 6 & 4,25 & 7,75 & 1,8 \\
\hline 62 & Falta de profesionalismo e implicación de colegios y sociedades profesionales. & 6,0 & 6 & 6 & 7 & 1,2 \\
\hline 63 & $\begin{array}{l}\text { Ausencia de capacidad y falta de valor para garantizar la estabilidad de las inversiones } \\
\text { del capital privado "serio". }\end{array}$ & 6,0 & 6 & 5,25 & 7 & 1,3 \\
\hline 64 & Falta coherencia entre la regulación del sistema y las necesidades-recursos & 5,6 & 6 & 4,25 & 7 & 1,6 \\
\hline 65 & $\begin{array}{l}\text { La insoportable levedad jurídica del SNS: sistema híbrido controlado por Hacienda y la } \\
\text { Seguridad Social. }\end{array}$ & 5,8 & 6 & 4 & 7 & 1,8 \\
\hline 66 & $\begin{array}{l}\text { Orientación de las agencias hacia la evaluación de tecnologías antes que hacia la evalua- } \\
\text { ción de servicios. }\end{array}$ & 5,5 & 6 & 3,5 & 7 & 2,0 \\
\hline 67 & Rechazo por parte de las asociaciones de pacientes que luchan por sus intereses particulares. & 5,9 & 6 & 5 & 6 & 1,2 \\
\hline 68 & Escaso nivel de formación y conocimientos técnicos de los diputados. & 5,3 & 6 & 4,25 & 6 & 1,4 \\
\hline 69 & $\begin{array}{l}\text { Conflicto de intereses (vínculos demasiado estrechos) entre las agencias de evaluación y } \\
\text { las administraciones sanitarias. }\end{array}$ & 5,7 & 5,5 & 4,25 & 7,75 & 1,8 \\
\hline 70 & Amplio abanico de prestaciones sanitarias: no se puede evaluar todo, ni hay estudios de todo. & 5,8 & 5,5 & 4 & 7,75 & 1,9 \\
\hline 71 & Escasa capacidad regulatoria de la administración en su ámbito. & 5,2 & 5,5 & 3,25 & 7 & 2,2 \\
\hline 72 & Escasa capacidad investigadora o evaluadora (baja masa crítica) en el SNS. & 5,3 & 5 & 4 & 6 & 1,5 \\
\hline 73 & $\begin{array}{l}\text { El diferente estado de salud de la población en cada área limita la aplicación de criterios } \\
\text { homogéneos. }\end{array}$ & 4,6 & 5 & 3 & 6 & 2,0 \\
\hline 74 & $\begin{array}{l}\text { Formación universitaria pregrado que limita el desarrollo curricular de aspectos básicos } \\
\text { sobre evaluación. }\end{array}$ & 5,1 & 4,5 & 3 & 7,75 & 2,6 \\
\hline 75 & El sistema está mas centrado en productos (visitas, actividad) que en servicios. & 4,8 & 4,5 & 2,25 & 7,5 & 3,3 \\
\hline 76 & Momento económico de fuertes restricciones presupuestarias. & 4,8 & 4 & 2,25 & 6 & 2,7 \\
\hline 77 & Hablar de "entes" (en lugar de redes u otras formas organizativas) puede ser una barrera. & 4,4 & 3 & 3 & 6 & 2,0 \\
\hline
\end{tabular}

SNS: Sistema Nacional de Salud; Med: Mediana: P: Percentil; RV: razón de variación entre el P75 y el P25.

NICE: National Institutes of Clinical Excellence. Las puntuaciones están ordenadas por mediana y media. 